\title{
Augmented Iterative Learning Control for Neural-Network-Based Joint Crest Factor Reduction and Digital Predistortion of Power Amplifiers
}

\author{
Siqi Wang, Morgan Roger, Member, IEEE, Julien Sarrazin, Senior Member, IEEE, and Caroline Lelandais-Perrault
}

\begin{abstract}
Digital predistorsion (DPD) is a commonly used approach to compensate for the power amplifiers (PA) nonlinearities and memory effects as well as to improve its power efficiency. To alleviate the restriction brought by high peak-to-average power ratio (PAPR) of input signals, crest factor reduction (CFR) is needed for higher efficiency. In modern communication systems, power of transmitted signals gets lower, which makes complexities of CFR and DPD become non-negligible. This paper proposes a new approach to realize a joint CFR and DPD model using neural networks (NN). The modeling accuracy is guaranteed by a new proposed augmented iterative learning control (AILC) algorithm for the NN training signals. Compared with conventional ILC, the proposed AILC is shown more robust according to simulation and experimental results. The proposed AILC-based NN-CFRDPD is experimentally evaluated on different test benches.
\end{abstract}

Index Terms-Crest factor reduction, digital predistortion, iterative learning control, neural networks, power amplifiers efficiency

\section{INTRODUCTION}

$\mathbf{M}$ ODERN green communications have stringent requirements on the power efficiency of power amplifiers (PA) which consume the majority of power in radio frequency (RF) telecommunication systems [1]. However, the nonlinearity of the PA causes spectral regrowth and introduces interference when the PA operating point is pushed towards saturation for high efficiency [2].

A common approach for PA linearization is digital predistortion (DPD), which has the inverse characteristics of the PA [3]. Applying the DPD upstream of the PA allows it working in its nonlinear zone, where the power efficiency is higher than in its linear zone [4]. Various DPD models have been developed, such as memory polynomial (MP) [5] based on Volterra series, generalized memory polynomial (GMP) [6], and dynamic-deviation-reduction (DDR) [7]. Block-oriented non linear (BONL) systems [8] have also been studied.

In modern communication systems, the peak-to-average power ratio (PAPR) of modulated signals, e.g. Orthogonal Frequency Division Multiplexing (OFDM) signals, brings another restriction on the PA efficiency. That is due to the necessary back-off on the PA operating point equal to or greater than the

Authors are with GeePs - Université Paris-Saclay, CentraleSupélec, CNRS, Laboratoire de Génie Electrique et Electronique de Paris, 91192, Gif-sur-Yvette, France. Sorbonne Université, CNRS, Laboratoire de Génie Electrique et Electronique de Paris, 75252, Paris, France (e-mail of authors: wangsiqi0807@gmail.com, morgan.roger@centralesupelec.fr, caroline.lelandais-perrault@centralesupelec.fr, julien.sarrazin@sorbonneuniversite.fr). signal PAPR, applied to avoid the PA saturation [9]. To reduce the signal PAPR, crest factor reduction (CFR) techniques can be implemented along with the DPD [10]. The clip-and-filter (CAF) approach is one of the most commonly used CFR approaches and is usually implemented upstream of the DPD [11]. The idea of CFR is to improve the PA efficiency while sacrificing some linearity if there is a margin beyond the lowest requirement of transmission standard after linearization by the DPD. Since it can also be implemented as a polynomial soft clipping as in [12], single models which combines the CFR and the DPD have been proposed in [13] [14].

The power consumption of the CFR and the DPD becomes crucial in modern wireless communication system, e.g. femtocell networks or 5G and beyond [15], especially for green communication [1]. It has been shown in [10] [14] that a DPD model can be identified using some certain techniques to realize also the function of a CFR, which improves the PA efficiency without additional complexity. This joint CFR/DPD model has been implemented with a conventional GMP model in [14] with a low complexity and an acceptable linearity. In this paper, we are seeking for a new joint CFR/DPD model using artificial neural networks (NN), which brings possibility to improve the linearization performance while trading off little complexity. Since better linearization performance renders larger linearization margin which allows more improvement of PA efficiency, the proposed NN-CFRDPD gives an alternative option in studying the trade-off between the power saved from the PA by the digital signal processing (DSP) and the power consumed by the DSP. Along with the development of low power-consuming digital circuits, e.g. especially the emerging memristor-based circuits for NN computation [16] [17], the computational consumption of the DSP is getting less and less significant, which makes the proposed NN-CFRDPD technique more and more promising.

In recent years, $\mathrm{NN}$ models have been largely used in different domains including the RF and microwave field [18]. In [19], a DPD model based on real-valued focused time-delay neural network (RVFTDNN) has been experimentally validated for PA linearization considering memory effects. Different NN models have been proposed to model the nonlinear behavior of wideband PAs and transmitters, such as two hidden layers artificial neural network (2HLANN) [20] [21], distributed spatiotemporal NN based model (DSTNN) [22], complex-Chebyshev functional link neural network (CCFLNN) [23]. The RVFTDNN has also been proposed in [24] to compensate simultaneously for the 
nonlinearity of the transmitters and the I/Q imbalance of the modulator. Furthermore, authors in [25] have additionally taken the crosstalk interference into consideration and proposed an NN model DPD for multi-input multi-output (MIMO) transmitters. In addition, the NN model can also be used to implement the CAF [26]. However, to the authors' knowledge, this paper is the first time where an $\mathrm{NN}$ is used for joint CFR/DPD.

The DPD parameter identification problem is traditionally dealt with direct learning architecture (DLA) or indirect learning architecture (ILA) [27]. However DLA has difficulties to train an NN-based DPD since the inverse function of an $\mathrm{NN}$ model is generally ill-defined. In [14], a joint CFR/DPD with GMP model has been identified using ILA. The reference signal for coefficients estimation is obtained thanks to clipping-and-bank-filtering technique [28]. In [29], authors advantageously applied the iterative learning control (ILC) algorithm to train an NN-based DPD for MIMO transmitters. The ILC is especially efficient in this case because it computes a target predistorted signal [4] [30]. However it fails to converge due to the added noise in the transmitted signals.

In this paper, we first propose an augmented ILC (AILC) algorithm which renders a target predistorted signal with better linearization performance compared with the conventional ILC. Indeed, the fact that additive white noise at the PA input is filtered iteratively in the ILC procedure generates out-ofband distortion because of the PA memory effects. This effect is analyzed and compensated in the proposed AILC procedure with a simple low pass filter. The proposed AILC enables the proposed NN-based joint CFR/DPD (NN-CFRDPD) to reach a satisfactory linearization performance while significantly enhancing the PA power efficiency. A simple two-hidden-layer time-delay (2HLTD) $\mathrm{NN}$ is used in this paper as the model. The proposed method is validated on different test benches. The proposed approach is compared with the approaches in the state-of-the-art. The comparison of linearization performance between the NN-CFRDPD with AILC and that with the conventional ILC confirms the advantages of our proposed method on the adjacent channel power ratio (ACPR) and the error vector magnitude (EVM).

This paper is organized as follows. Section II presents the conventional iterative learning control algorithm, its limitations, and the augmented version we propose. Section III gives simulation results of the proposed AILC with different noise levels. The structure and training process of the proposed NN-CFRDPD are then described in Section IV. In Section $\mathrm{V}$, the test bench and the corresponding experimental results are presented and discussed. Finally, the conclusion is given in Section VI.

\section{Augmented Iterative LEARNING CONTROL}

ILC adjusts the PA input signal in an iterative procedure so that the PA output signal converges towards a desired signal. Without the need to know the DPD model structure, a desired predistorted signal at the PA input is obtained after iterations [31].

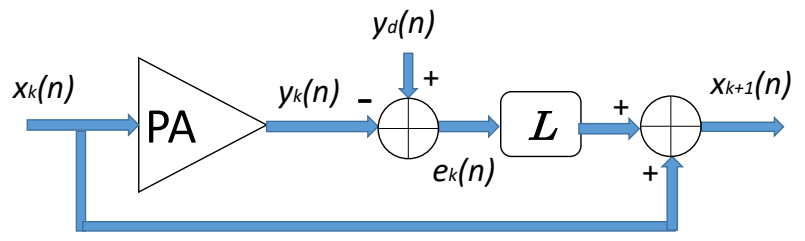

Fig. 1. Process of the $k$-th iteration in ILC

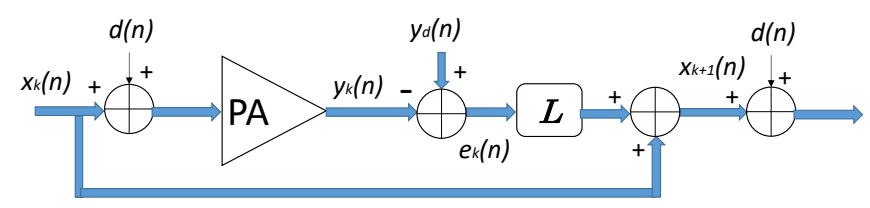

Fig. 2. ILC scheme with noised PA input signal at the $k$-th iteration

\section{A. Conventional ILC for PA linearization}

The ILC scheme is depicted in Fig. 1. The PA input and output signals at the $k$-th iteration are denoted by $x_{k}(n)$ and $y_{k}(n) \cdot y_{d}(n)$ is the desired PA output signal and $e_{k}(n)$ represents the observed error between the desired signal and the measured PA output:

$$
e_{k}(n)=y_{d}(n)-y_{k}(n) .
$$

At the first iteration, the PA input is initialized with the stimulus of the system. At the $k$ th iteration, a new PA input according to the observed error $e_{k}(n)$ is computed:

$$
\boldsymbol{x}_{k+1}=\boldsymbol{x}_{k}+\mathbf{L} \boldsymbol{e}_{k},
$$

where $\boldsymbol{x}_{k}=\left[x_{k}(1), \ldots, x_{k}(N)\right], \boldsymbol{e}_{k}=\left[e_{k}(1), \ldots, e_{k}(N)\right], N$ is the number of signal samples, $\mathbf{L}$ is the learning matrix. In [4], a linear ILC is proposed by setting the learning matrix $\mathbf{L}=\gamma \mathbf{I}$, where $0<\gamma<\frac{2}{G_{s s}}$ and $G_{s s}$ is the PA small signal gain [32]. Then eq. 2 can be rewritten as

$$
x_{k+1}(n)=x_{k}(n)+\gamma e_{k}(n) .
$$

The procedure ends when the error $e_{k}(n)$ meets the chosen stop condition.

However, the convergence stays in level of ideal system without noise which has a dramatic impact. To improve the bound of ILC performance, we need to take the noise into consideration as in next section.

\section{B. Iterative filtering effect}

With real measurements, it is unavoidable to bring noise into the PA input signal during digital-to-analog conversion and RF modulation as illustrated in Fig. 2, where $d(n)$ is Gaussian white noise (GWN).

The distortion of the PA is mainly due to its nonlinearity and memory effects. Since the power of the added noise $d(n)$ is equally distributed at all frequencies and is weak compared with the useful signal power, it is easy to know that $d(n)^{k}$ is also GWN distributed at all frequencies. Therefore, the PA nonlinearity has very few impact on it. However, the memory 


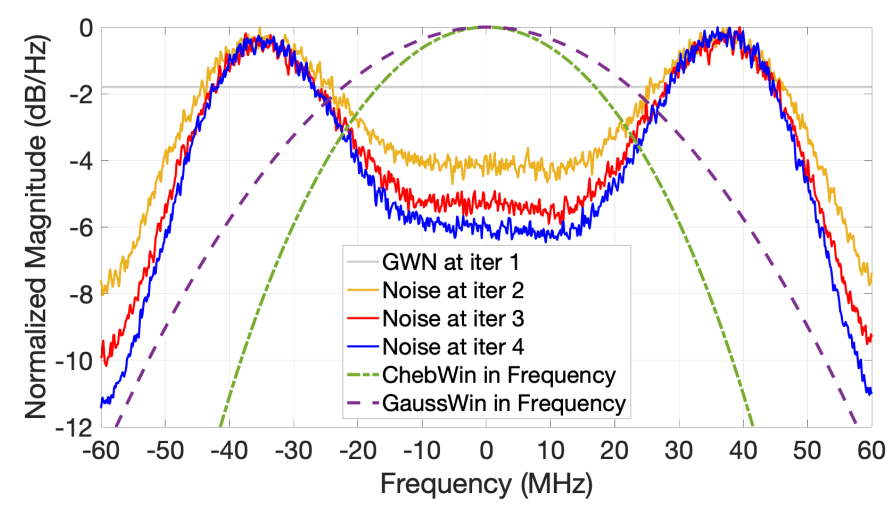

Fig. 3. Noise after iterative filtering and some frequency windows for equalization

effects of the PA have impact on the out-of-band distortion at frequencies away from the useful signal band [33]. Due to memory effects, the output signal of a PA depends on both the present and the historical input signal samples. Thus a linear filter can be used to model the PA memory effects in baseband digital signal processing [34]:

$$
s_{\text {out }}(n)=\sum_{l=0}^{L} c_{l} s_{\text {in }}(n-l),
$$

where $s_{\text {in }}$ and $s_{\text {out }}$ are the filter input and output signals respectively, $L$ is the memory depth, $c_{l}$ are the filter coefficients. If we express the noise component at frequency $f$ as

$$
d_{f}(n)=A e^{j 2 \pi f n t},
$$

where $t=1 / f_{s}$ with sampling frequency $f_{s}, A$ is the amplitude, the filtered noise becomes

$$
\begin{aligned}
d_{\nu}^{F}(n) & =A \sum_{l=0}^{L} c_{l} e^{j 2 \pi \nu(n-l)} \\
& =\left(\sum_{l=0}^{L} c_{l} e^{-j 2 \pi \nu l}\right) d_{\nu}(n),
\end{aligned}
$$

where $\nu=f / f_{s}$ represents the normalized frequency. The amplitude of the filtered noise component $d_{\nu}^{F}(n)$ is a function of the normalized frequency $\nu$. In other words, the filtered noise $d^{F}(n)$ is no longer white noise.

Since ILC is an iterative procedure, the noise $d(n)$ is filtered iteratively and is accumulated in $e(n)$. The noise at $k$-th iteration can be expressed as

$$
\begin{array}{r}
d_{\nu, k}(n)=d_{\nu}(n)+M(\nu) d_{\nu, k-1}(n), \\
\text { where } M(\nu)=\sum_{l=0}^{L} c_{l} e^{-j 2 \pi \nu l} .
\end{array}
$$

After $K$ iterations, the iteratively filtered (IF) noise can be approximated to

$$
d_{\nu, K}^{\mathrm{IF}}(n)=\sum_{k=0}^{K} M^{k}(\nu) d_{\nu}(n) .
$$

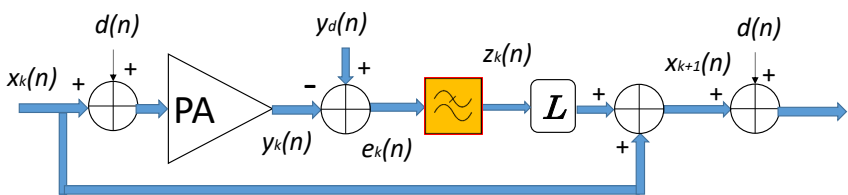

Fig. 4. AILC scheme at the $k$-th iteration

We denote the iterative filtering gain by

$$
G_{K}^{\mathrm{IF}}(\nu)=\left|\sum_{k=0}^{K} M^{k}(\nu)\right| .
$$

At the frequencies where $G_{K}^{\mathrm{IF}}(\nu)>1$, the noise is amplified as shown in Fig. 3 and the convergence of ILC is influenced.

The spectral evolution of GWN for 4 iterations is depicted in Fig. 3. To equalize the amplified noise, we propose to use some frequency windows, such as Chebyshev window and Gaussian window, to filter it.

\section{AILC with spectral windowing correction}

The iteratively filtered noise components in the band of $y_{d}(n)$ are negligible compared with the power of $y_{d}(n)$ and can be compensated by (1). We propose an augmented version of ILC to equalize the iteratively filtered noise components at out-of-band frequencies with windows in the frequency domain as illustrated in Fig. 3.

The AILC scheme is depicted in Fig. 4. At the $k$-th iteration, on the error $e_{k}(n)$ obtained by (1), we apply a window in the frequency domain to filter the out-of-band components of $e_{k}(n)$ :

$$
\mathcal{F}\left\{z_{k}(n)\right\}(\nu)=\mathcal{F}\left\{d_{k}\right\}(\nu) \cdot W(\nu)
$$

where $\mathcal{F}\{\cdot\}$ represents the discrete Fourier transform of $N_{\mathrm{FT}}$ samples, $W$ is a window centered at frequency 0 in baseband with length $N_{\mathrm{FT}}$, and $z_{k}(n)$ is the windowed observed error of AILC. The window $W$ is constant at each iteration. The values of $W$ are real values with $\max (W)=1$ and $\min (W)=0$. The width of the window is determined by the sampling frequency of the system. The spectra of Gaussian frequency window and Chebyshev frequency window are depicted in Fig. 3. Chebyshev window has steeper descent at high frequency.

The window is an equalizer for the iteratively filtered gain $G_{1}^{\mathrm{IF}}(\nu)$ especially when $G_{1}^{\mathrm{IF}}(\nu)$ increases along with $\nu$ increasing.

\section{Simulation RESUlts of AILC}

In order to validate the mathematical derivation of amplified noise in the previous section, we feed an LTE signal noised by different levels of GWN to a PA model.

In this section, we use a Wiener model PA [35] with a $20 \mathrm{MHz}$ LTE signal for baseband simulation comparison between the conventional ILC and the proposed AILC. The AILC is implemented with either a Gaussian window or a Chebyshev window. The Chebyshev window is applied with $100 \mathrm{~dB}$ of sidelobe attenuation. 


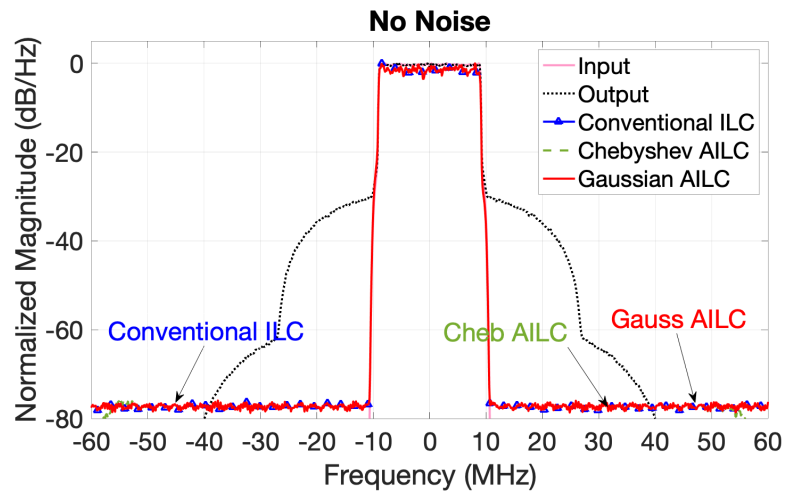

Fig. 5. simulation of conventional ILC and AILC without noise

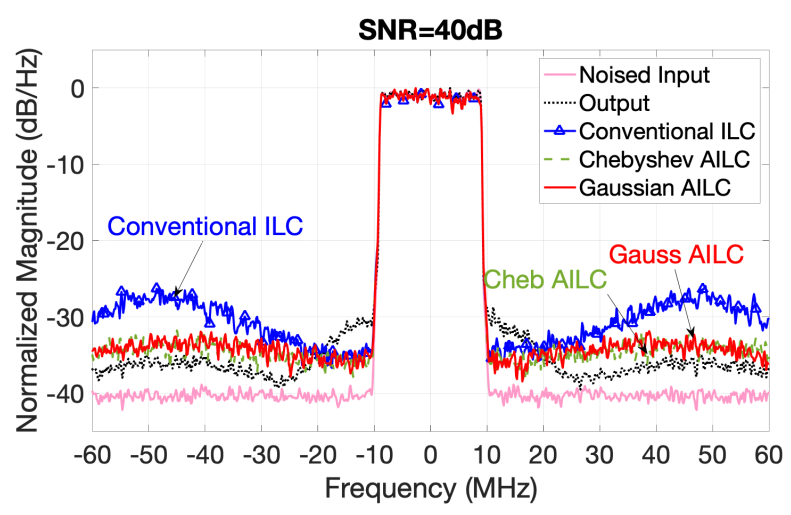

Fig. 6. simulation of conventional ILC and AILC with $\mathrm{SNR}=40 \mathrm{~dB}$

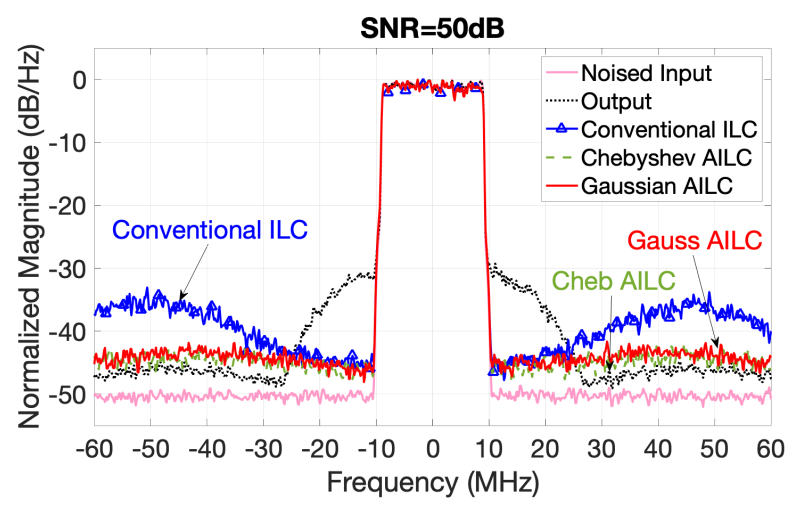

Fig. 7. simulation of conventional ILC and AILC with $\mathrm{SNR}=50 \mathrm{~dB}$

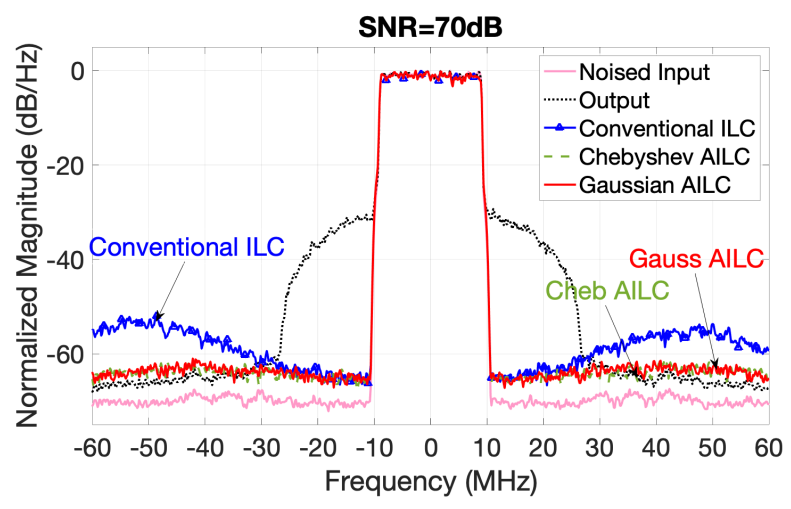

Fig. 8. simulation of conventional ILC and AILC with SNR=70dB
TABLE I

AILC VS ILC WITH DIFFERENT SNR

\begin{tabular}{|l|c|c|c|c|}
\hline \multicolumn{2}{|c|}{} & $\begin{array}{c}\text { Conventional } \\
\text { ILC }\end{array}$ & $\begin{array}{c}\text { Chebyshev } \\
\text { AILC }\end{array}$ & $\begin{array}{c}\text { Gaussian } \\
\text { AILC }\end{array}$ \\
\hline \multirow{3}{*}{$\begin{array}{l}\text { No noise } \\
\text { ACPR }(d B c)\end{array}$} & L1 & -75.4 & -75.4 & -75.4 \\
\cline { 2 - 5 } & U1 & -75.4 & -75.4 & -75.4 \\
\cline { 2 - 5 } & L2 & -75.4 & -75.3 & -75.4 \\
\cline { 2 - 5 } & U2 & -75.4 & -75.5 & -75.4 \\
\hline \multirow{3}{*}{$\begin{array}{l}\text { SNR=70dB } \\
\text { ACPR }(d B c)\end{array}$} & L1 & -61.5 & -63.3 & -62.8 \\
\cline { 2 - 5 } & U1 & -60.6 & -63.0 & -62.9 \\
\cline { 2 - 5 } & L2 & -53.4 & -61.9 & -61.5 \\
\cline { 2 - 5 } & U2 & -54.4 & -61.9 & -61.5 \\
\hline \multirow{3}{*}{$\begin{array}{l}\text { SNR=50dB } \\
\text { ACPR }(d B c)\end{array}$} & U1 & -41.9 & -43.9 & -43.4 \\
\cline { 2 - 5 } & L2 & -41.0 & -43.8 & -43.1 \\
\cline { 2 - 5 } & U2 & -34.8 & -42.6 & -41.9 \\
\hline \multirow{3}{*}{$\begin{array}{l}\text { SNR=40dB } \\
\text { ACPR }(d B c)\end{array}$} & L1 & -31.8 & -42.6 & -41.9 \\
\cline { 2 - 5 } & U1 & -31.6 & -33.8 & -33.4 \\
\cline { 2 - 5 } & U2 & -26.5 & -33.6 & -33.3 \\
\cline { 2 - 5 } & & -26.6 & -32.4 & -31.9 \\
\hline
\end{tabular}

By launching the conventional ILC and the proposed AILC algorithms with different noise levels, we get the corresponding PA output $y_{k}(n)$ and plot their spectra for comparison in Fig. 5-8. We first test these methods without additive noise and verify that the conventional ILC can converge in this ideal case as depicted in Fig. 5. The AILC is the same as the conventional ILC, which can reach the best performance. Then we add white noise to the PA input signal (Noised input) with the signalto-noise ratio (SNR) equal to $40 \mathrm{~dB}, 50 \mathrm{~dB}$ and $70 \mathrm{~dB}$. The corresponding PA output spectra are illustrated in Fig. 6, Fig. 7 and Fig. 8 respectively.

In these tests, iterations stop when the error power $\sum_{n=1}^{N}\left|e_{k}(n)\right|^{2}$ does not decrease. The ACPR values in the first/second lower/upper (L/U) adjacent channels are listed in Table I. If we denote the stimulus bandwidth by $B$, the first lower/upper (L1/U1) adjacent channels are $[-3 B / 2,-B / 2]$ and $[B / 2,3 B / 2]$ respectively, the second lower/upper (L2/U2) adjacent channels are $[-5 B / 2,-3 B / 2]$ and $[3 B / 2,5 B / 2]$.

With the noise added at the PA input, we can see that the conventional ILC has spectral regrowth at out-of-band frequencies as predicted in Section II-B. The proposed AILC improves the ACPR around $2 \mathrm{~dB}$ in the first band and more than $5 \mathrm{~dB}$ in the second band. Furthermore the AILC with Chebyshev window shows better performance than Gaussian window. The experimental confirmation will then be given in Section V.

\section{NEURAL NETWORK-BASED JOINT CFR/DPD}

This section presents the NN architecture and details the training methodology. The training signals are obtained by the proposed AILC. The stimulus $u(n)$ is the input of NN and the desired predistorted signal given by AILC is the target signal of NN. Since the NN output cannot be better than the target signal, its linearization performance is bounded by the AILC. With the conventional ILC, the performance of the NN model is heavily restricted due to the noised error. With the proposed AILC, the bound is lowered and this allows the NN to achieve better linearization performance.

In order to realize the function of CFR in the NN model, the target signal generated by the AILC procedure presented in 


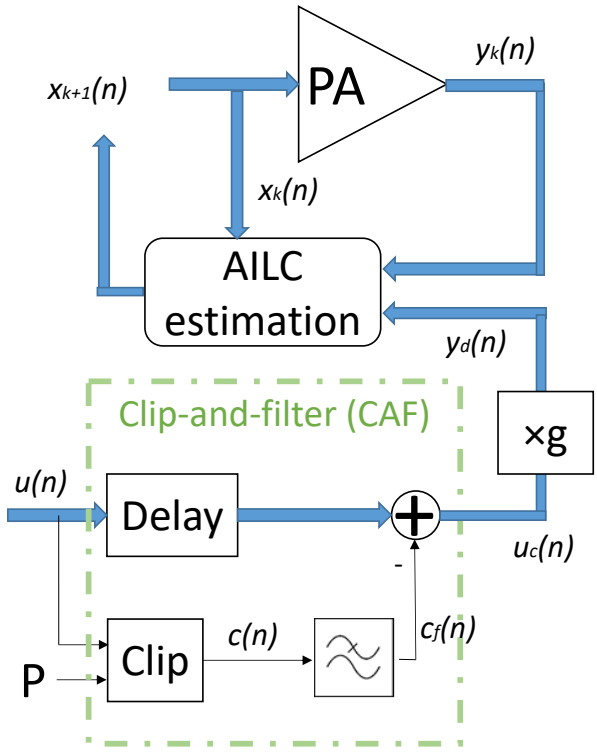

Fig. 9. Clipping $u(n)$ for desired signal $y_{d}(n)$ in AILC

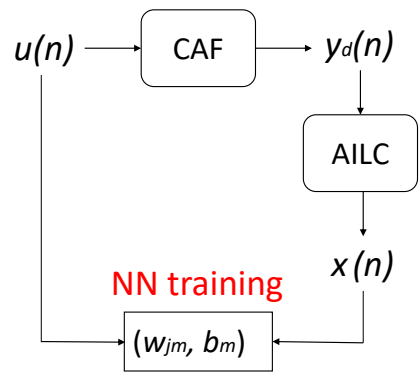

Fig. 10. Procedure of NN-CFRDPD training

Section II-C should result in a clipped linearized signal $y_{d}(n)$ at the PA output. Simply taking the stimulus $u(n)$ multiplied by the PA gain $g$ as $y_{d}(n)$ as in [4] can only give a predistorted signal for PA linearization. In this paper, we take a clipped signal $u_{c}(n)$ with reduced PAPR for the desired $y_{d}(n)$ as illustrated in Fig. 9.

In this paper, we compute $u_{c}(n)$ using traditional clipand-filter (CAF) [10]. The CAF is composed of mainly two steps. First, it clips the signal samples which exceed the given threshold $P$ to obtain a correction signal

$$
c(n)= \begin{cases}u(n)\left(1-\frac{P}{|u(n)|}\right) & \text { if }|u(n)| \geq P, \\ 0 & \text { otherwise, }\end{cases}
$$

Then, we apply a filter with the same bandwidth as $u(n)$ on $c(n)$ to remove the out-of-band frequency components. The clipping step may generate new peaks and thus we repeat for a certain number of iterations to obtain a final correction signal $c_{f}(n)$ [36]. We use the signal clipped by the CAF approach to compute the desired signal $y_{d}(n)$ of AILC

$$
y_{d}(n)=g \cdot u_{c}(n)=g \cdot\left(u(n)-c_{f}(n)\right)
$$

where $g$ is the gain of the PA. This desired signal is then used in AILC to compute the NN target signal $x(n)$ by (1).

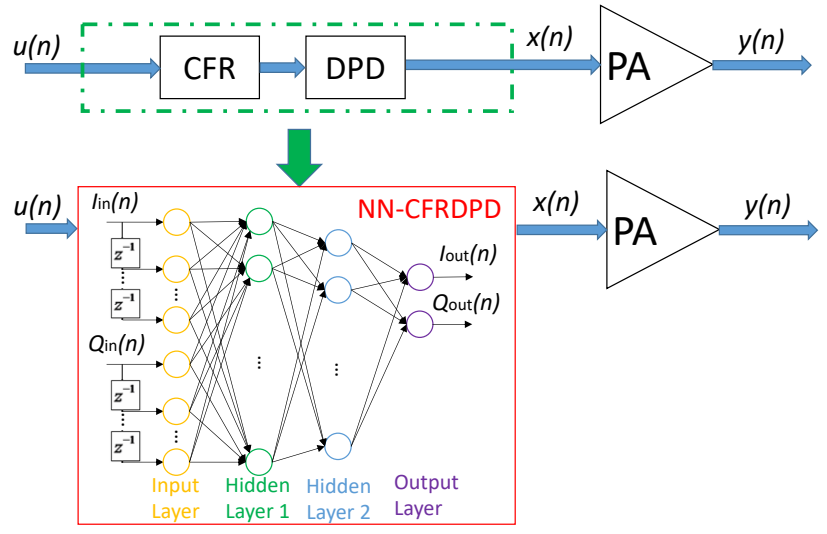

Fig. 11. The structure of NN-CFRDPD

The entire identification procedure of NN-CFRDPD is summarized in Fig. 10. It can be decomposed into three steps:

1) Calculating desired signal $y_{d}(n)$ for AILC with $u(n)$.

2) Estimating the target signal $x(n)$ which is the predistorted clipped signal obtained by AILC.

3) Training the NN model with $u(n)$ and $x(n)$.

The idea of the NN-CFRDPD structure is illustrated in Fig. 11. The NN-CFRDPD is equivalent to the cascade of the CFR and the DPD, which means the NN input is the stimulus $u(n)$ and its output is the PA input signal $x(n)$. The PA output signal is denoted by $y(n)$. In the 2HLTDNN, the input and output are decomposed into real-valued I and $\mathrm{Q}$ signals:

$$
\begin{aligned}
& u(n)=I_{\text {in }}(n)+i Q_{\text {in }}(n) \\
& x(n)=I_{\text {out }}(n)+i Q_{\text {out }}(n) .
\end{aligned}
$$

$I_{\text {in }}(n), Q_{\text {in }}(n)$ and their $L$ delayed samples construct the input layer (yellow circles in Fig. 11). The output layer has only two neurons: $I_{\text {out }}(n)$ and $Q_{\text {out }}(n)$. Between the input and output layers, there are two hidden layers as the green and blue circles show in Fig. 11. The number of neurons in the $l$-th hidden layer is denoted by $N_{l}$. The output $q_{m l}$ of the $m$-th neuron in the $l$-th layer is a function of its inputs $t_{j}$ :

$$
q_{m(l)}=f\left(\sum_{j=1}^{N_{l-1}} w_{j m} t_{j}+b_{m}\right)
$$

where $w_{j m}$ is the weight, $b_{m}$ is the bias and $f(\cdot)$ is the activation function. In this paper, we chose the rectified linear unit (ReLU) as activation function for hidden layers [37]:

$$
\operatorname{ReLU}(x)= \begin{cases}x & \text { if } x \geq 0 \\ 0 & \text { otherwise }\end{cases}
$$

The activation function of the output layer is linear.

Knowing the target signal $x(n)$, the $\mathrm{NN}$ can be trained and its weights computed by backward computation using the Levenberg-Marquardt algorithm [38]. We randomly divide the input and target signals into 3 segments for training, validating and testing. The process can be composed of 2 phases:

1) At each epoch, we take the first segment to compute the values of $w_{j m}$ and $b_{m}$ and validate the computed NN with the second segment. 


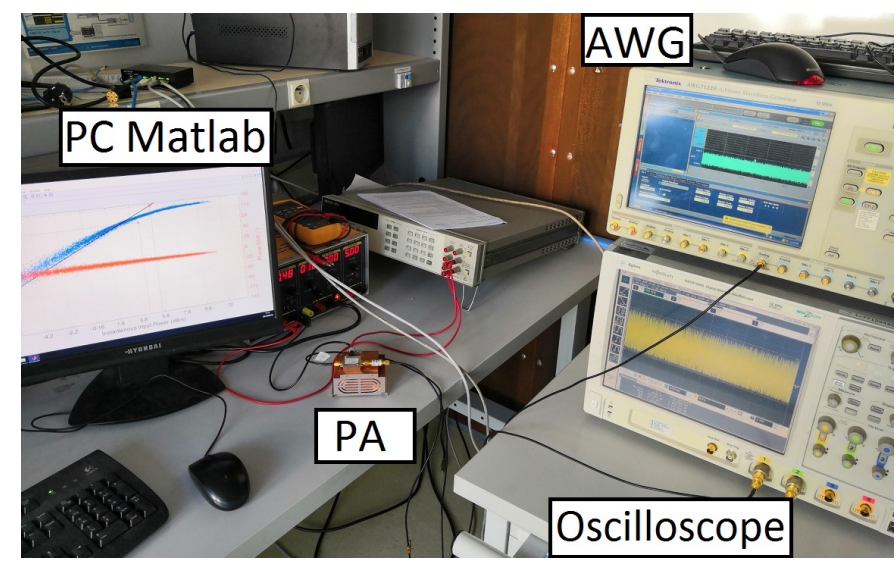

Fig. 12. Test bench for Experimental Implementation

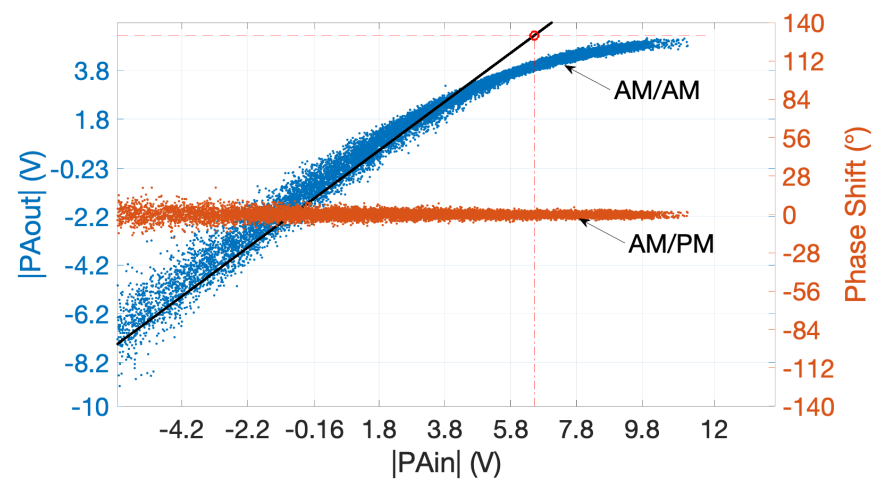

Fig. 13. AMAM \& AMPM curves of Test bench 1

2) After the training, we test the NN with the third segment.

In validating and testing step, the mean square error (MSE) between the target signal and the NN output signal is calculated to estimate the NN modeling accuracy. The maximum training epochs are set to 100 .

\section{EXPERIMENTAL RESULTS}

\section{A. Test bench}

In this section, we give experimental results using two test benches.

1) Test bench 1: The first test bench is illustrated in Fig. 12 . The device under test (DUT) is a HMC409LP4E PA operating in the 3.3-3.8 GHz band. Its nominal gain at $3.5 \mathrm{GHz}$ is $31 \mathrm{~dB}$ and the saturated output power is $32.5 \mathrm{dBm}$. The supply voltage is $5 \mathrm{~V}$. The AM/AM \& AM/PM (Amplitude Modulation/Amplitude Modulation \& Amplitude Modulation/Phase Modulation) curves is depicted in Fig. 13. We generate the baseband stimulus in the PC workstation and feed it to the PA through an Arbitrary Waveform Generator (AWG) at $10 \mathrm{GHz}$ sampling frequency. The AWG generates the stimulus directly at the $3.5 \mathrm{GHz}$ carrier frequency and no external mixer are therefore used. The PA output signal is directly captured by an oscilloscope with sampling rate at $10 \mathrm{GHz}$ and is fed back to the PC workstation for postdistortion processing. The input and output baseband signals are synchronized in time after down-sampling to $200 \mathrm{MHz}$ for the NN-CFRDPD training.

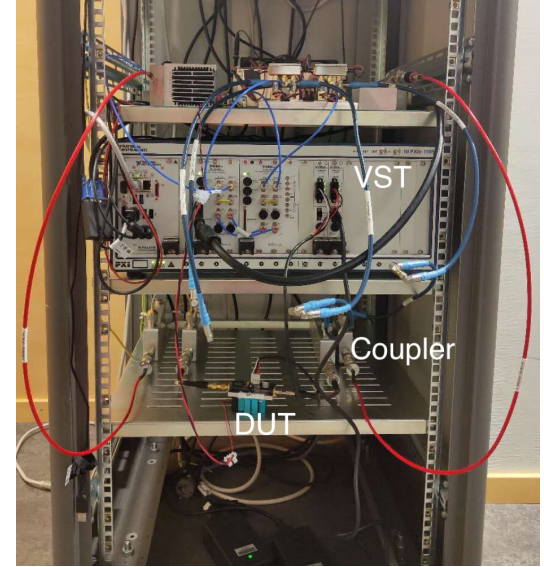

Fig. 14. Test bench of WebLab [39] for Experimental Implementation.

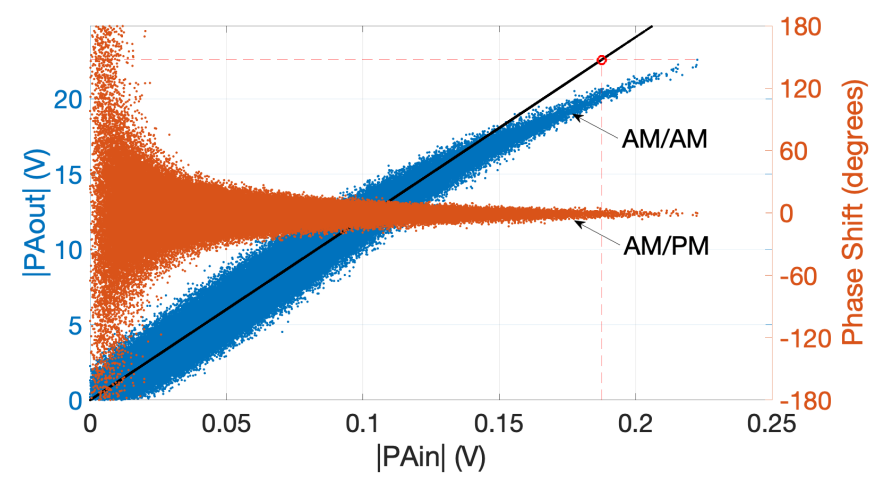

Fig. 15. AMAM \& AMPM curves of Test bench 2 (WebLab)

2) Test bench 2: We use test bench of WebLab [39] for measurements as depicted in Fig. 14. The baseband IQ signal is fed from the PC Workstation to the driver through a Vector Signal Transceiver (PXIe-5646R VST) using a $200 \mathrm{MHz}$ sampling frequency. The supply voltage is $5 \mathrm{~V}$. The VST up-converts the baseband signal to the carrier frequency $2.14 \mathrm{GHz}$. The signal at the output of the PA is then downconverted to baseband by the VST which provides to the PC workstation the baseband signal digitized with a sampling frequency of $200 \mathrm{MHz}$. A GaN PA CGH40006P transistor mounted in the manufacturer demo-board fabricated by CREE has been used to validate the proposed low rate DPD. Its nominal gain is $13 \mathrm{~dB}$ at $2 \mathrm{GHz}$ and the output power at $1 \mathrm{~dB}$ gain compression is $40.2 \mathrm{dBm}$. The nonlinearities and the memory effect of this PA can be seen from the AMAM\&AMPM curves in Fig 15 in the case the stimulus is $60 \mathrm{MHz}$ LTE signal. The average power of the signal at the input of the driver is around $-24.94 \mathrm{dBm}$. The measured average output power of the PA is $27.43 \mathrm{dBm}$.

\section{B. $A I L C$}

In section III, we have shown that our proposed AILC outperforms the conventional ILC by simulation. In this section, we experimentally test the conventional ILC and the proposed AILC to confirm that our proposed AILC has tackled the problem of convergence which can significantly improve the linearization performance. We compute the target signal $x(n)$ 


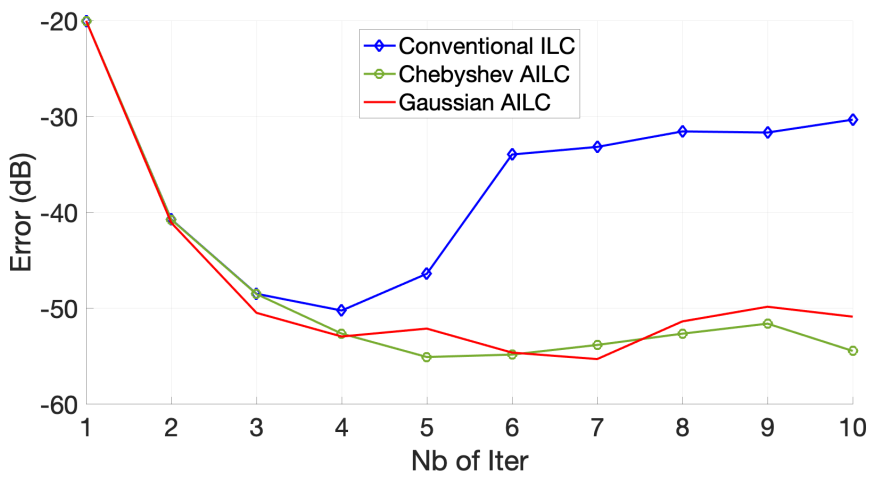

Fig. 16. Error convergence comparison of conventional ILC and AILC

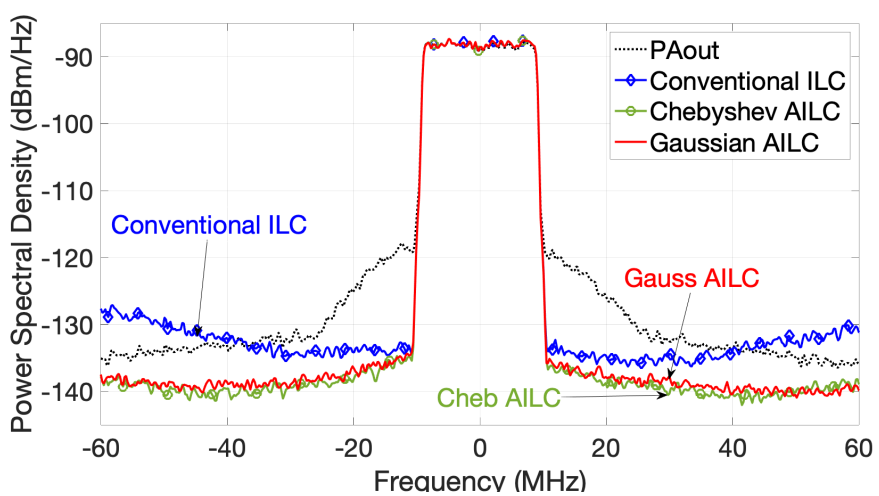

Fig. 17. PA output spectra with conventional ILC and different AILC

for NN training using conventional ILC and the proposed AILC algorithm.

With Test bench 1, Fig. 16 gives the error convergence of conventional ILC and AILC when the stop condition is set to 10 iterations. The error in $\mathrm{dB}$ is calculated by

$$
E_{\mathrm{dB}}=10 \log _{10}\left(\frac{1}{N} \sum_{n=1}^{N}|e(n)|^{2}\right) .
$$

We can see that the error of conventional ILC is worse than AILC after 3 iterations and it diverges after 4 iterations. The error of our proposed AILC converges to $-55 \mathrm{~dB}$ after 5 iterations. Compared with Gaussian window, the error of the Chebyshev window AILC is higher at the 3rd iteration but lower at the 5th iteration. With Chebyshev window, the proposed AILC converges more slowly but more steadily.

While implementing the AILC, we set the stop condition as $E_{\mathrm{dB}}^{(k)} \geqslant E_{\mathrm{dB}}^{(k-1)}$. The linearized PA output spectra are illustrated in Fig. 17. The gray dotted curve is the PA out-

TABLE II

COMPARISON OF DIFFERENT METHODS WITH TEST BENCH 1

\begin{tabular}{|l|c|c|c|c|}
\hline \multicolumn{2}{|c|}{} & $\begin{array}{c}\text { Conventional } \\
\text { ILC }\end{array}$ & $\begin{array}{c}\text { Chebyshev } \\
\text { AILC }\end{array}$ & $\begin{array}{c}\text { Gaussian } \\
\text { AILC }\end{array}$ \\
\hline \multirow{3}{*}{$\begin{array}{c}\text { ACPR } \\
(\mathrm{dBc})\end{array}$} & L1 & -44.1 & -48.6 & -47.4 \\
\cline { 2 - 5 } & U1 & -45.1 & -48.1 & -47.9 \\
\cline { 2 - 5 } & L2 & -41.4 & -50.1 & -49.1 \\
\cline { 2 - 5 } & U2 & -43.4 & -50.5 & -49.9 \\
\hline \multicolumn{2}{|c|}{ EVM (\%) } & 4.9 & 3.2 & 3.6 \\
\hline
\end{tabular}

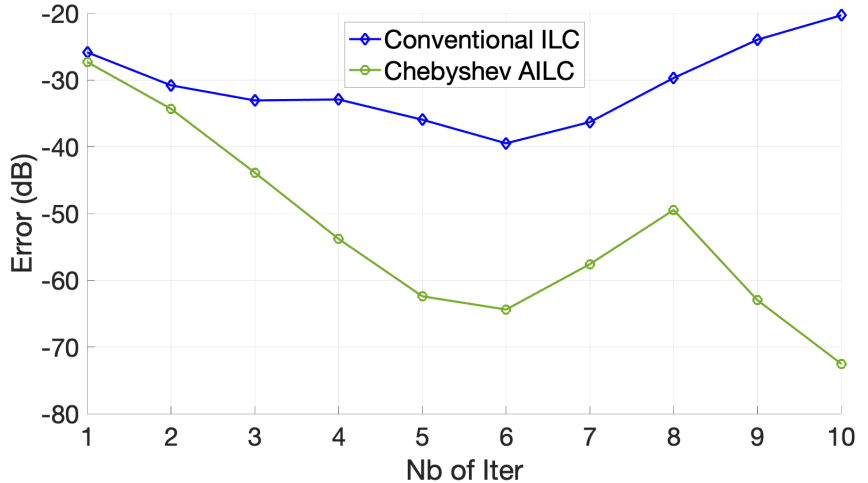

Fig. 18. Error convergence comparison of conventional ILC and AILC

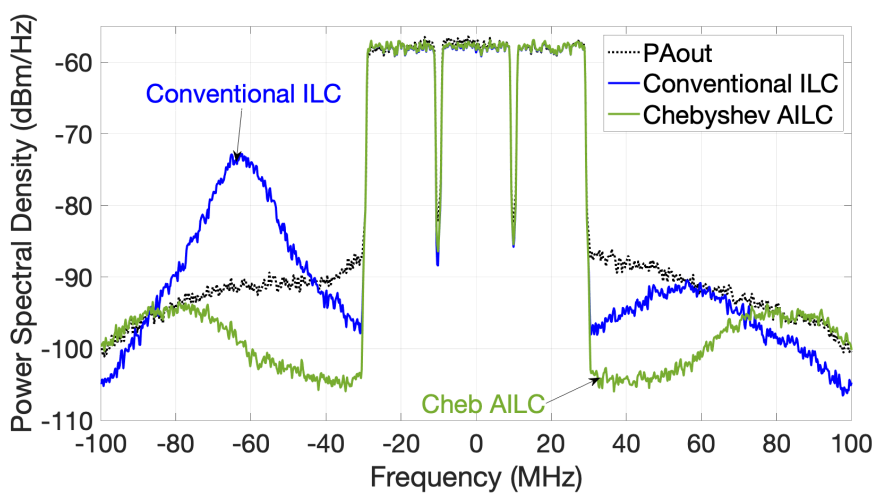

Fig. 19. PA output spectra with conventional ILC and different AILC

put without linearization. The blue diamond curve represents the conventional ILC. The green circle curve represents the Chebyshev AILC. The red curve represents the Gaussian AILC. The ACPR values are listed in Table II.

These experimental results validate the robustness of the proposed AILC. We can see that our proposed AILC has advantage beyond the conventional ILC about $3 \mathrm{~dB}$ in the first band and over $6 \mathrm{~dB}$ in the second band. The performance of Chebyshev AILC is about $1 \mathrm{~dB}$ better than that of Gaussian AILC. This corresponds to the curves in Fig. 16. With the current stop condition, Gaussian AILC stops at the 4th iteration while Chebyshev AILC stops at the 5th iteration.

Using Test bench 2, we can also observe that the problem of convergence is tackled by using AILC as illustrated in Fig. 18 and Fig. 19. Since AILC with Chebyshev window is found better according to the results with Test bench 1 , we test only Chebyshev AILC with Test bench 2 . The linearization performance is listed in Table III. In this case, the proposed AILC achieves more than $10 \mathrm{~dB}$ improvement on ACPR compared with ILC.

TABLE III COMPARISON OF DIFFERENT METHODS WITH TEST BENCH 2

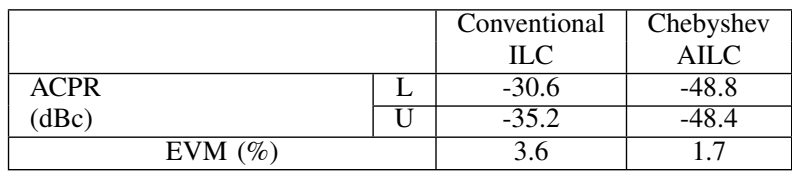


TABLE IV

NUMBER OF FLOPS FOR OPERATIONS

\begin{tabular}{|l|c|l|c|}
\hline Operation & flops & Operation & flops \\
\hline Real Addition & 1 & Real Multiplication & 1 \\
\hline Real Division & 4 & Complex Addition & 2 \\
\hline Complex Multiplication & 6 & $\begin{array}{l}\text { Complex-Real } \\
\text { Sultiplication }\end{array}$ & 2 \\
\hline Square-root & $6 \sim 8$ & Mution & 2 \\
\hline
\end{tabular}

With the proposed AILC, we can obtain a better target signal for the NN-CFRDPD training, which may improve the linearization margin and thus gives more possibility to enhance the PA efficiency. In case of Test bench 2, using AILC to estimate the target signal for NN-CFRDPD training is indispensable.

\section{Complexity Analysis}

Since the identification of models is not required all the time, in this paper we consider only running complexity [40]. We estimate the complexity with the number of flops (floatingpoint operation) for each sample. Table IV gives the number of flops of each operation according to [40]. Since different algorithms are available for the square-root operation, the number of flops is around 6 8. We take the average 7 flops in this paper.

According to [10] [14], the joint CFR/DPD has obvious advantage on complexity and linearization performance as well compared with the traditional CFR algorithm which is implemented independently of the DPD. Therefore we mainly focus on comparing the complexities of GMP-based CFRDPD in [21] and the proposed NN-CFRDPD.

1) GMP complexity estimation: The GMP model [6] is expressed as

$$
\begin{aligned}
x(n)= & \sum_{k=0}^{K_{a}} \sum_{l=0}^{L_{a}} \theta_{a, k l} u(n-l)|u(n-l)|^{k} \\
& +\sum_{k=1}^{K_{b}} \sum_{l=0}^{L_{b}} \sum_{m=1}^{M_{b}} \theta_{b, k l m} u(n-l)|u(n-l-m)|^{k} \\
& +\sum_{k=1}^{K_{c}} \sum_{l=0}^{L_{c}} \sum_{m=1}^{M_{c}} \theta_{c, k l m} u(n-l)|u(n-l+m)|^{k} .
\end{aligned}
$$

where $k$ is the index for nonlinearity, and $l, m$ are the indices for memory. The vector $\theta=\left[\theta_{a}, \theta_{b}, \theta_{c}\right]$ contains the complex coefficients for the signal and envelope, the signal and lagging envelope, and the signal and leading envelope, respectively.

According to [40], the running complexity of a GMP model for each sample signal can be expressed as

$$
F_{\mathrm{GMP}}=8 R-2
$$

where $R$ is the number of model coefficients.

2) NN complexity estimation: The inputs of $\mathrm{NN}$ are real values. In eq. (14), there are $N_{l-1}$ real multiplications and $N_{l-1}+1$ summation. Since ReLU does only a comparison, we can neglect its complexity. Thus the number of flops for 2-hidden-layer $\mathrm{NN}$ is

$$
F_{\mathrm{NN}}=(4 L+1) N_{1}+\left(2 N_{1}+1\right) N_{2}+2\left(2 N_{2}+1\right)
$$

where $L$ is the memory depth, $N_{i}$ is number of neurons in the $i$-th layer.

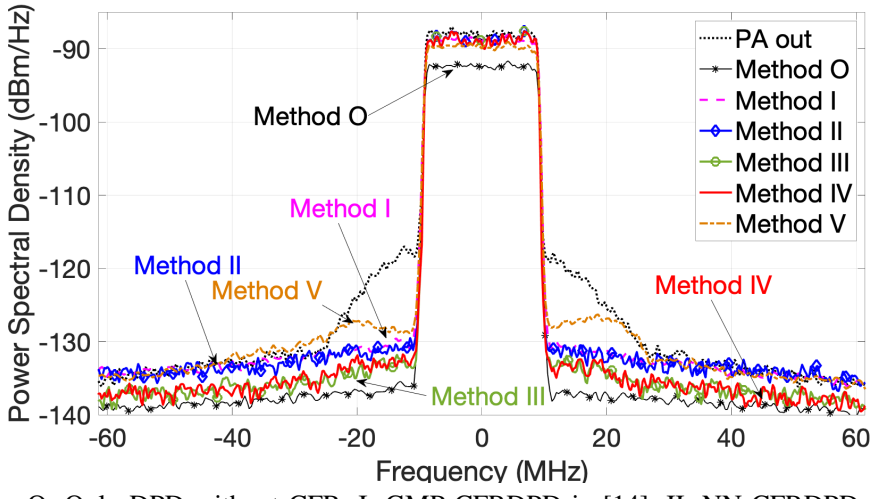

O: Only DPD without CFR; I: GMP-CFRDPD in [14]; II: NN-CFRDPD with conventional ILC; III: NN-CFRDPD with Chebyshev AILC; IV: NN-CFRDPD with Gaussian AILC V: GMP-CFRDPD in [13];

Fig. 20. PA output spectra without and with only DPD, and with NNCFRDPD on Test bench 1

\section{NN-CFRDPD}

With the target signal obtained by the proposed AILC, an NN-CFRDPD is trained and implemented in this section. We take 50000 samples from the stimulus and the predistorted signal obtained in Section V-B as the target signal to train the NN-CFRDPD. We randomly select $70 \%$ samples for training step, $15 \%$ samples for validating step and $15 \%$ samples for testing step. We set NN model with time delay $L=6$ and $N_{1}=N_{2}=30$ neurons in each hidden layer for all tests. These hyperparameters are determined by the algorithm proposed in [21]. The proposed NN-CFRDPD is then validated on the test bench with another segment of signal with 40000 samples.

First we test our proposed AILC-based NN-CFRDPD on Test bench 1 and compare it with approaches in the stateof-the-art. We take a generalized memory polynomial model (GMP) [6] for a joint CFR/DPD [14] as references. According to the algorithm in [41], we have the DPD model structure

$$
\begin{aligned}
\mathcal{K}_{a} & =2, \mathcal{L}_{a}=4 \\
\mathcal{K}_{b} & =1, \mathcal{L}_{b}=1, \mathcal{M}_{b}=1 \\
\mathcal{K}_{c} & =6, \mathcal{L}_{c}=2, \mathcal{M}_{c}=1
\end{aligned}
$$

with 21 coefficients.

The PA output spectra linearized with different methods are illustrated in Fig. 20. The gray dotted curve is the PA output without DPD linearization. The black point curve stands for the PA output linearized by only DPD (method O). The pink dashed curve represents the GMP-based joint CFR/DPD in [14] (method I). The blue diamond curve represents the conventional-ILC-based NN-CFRDPD method (method II). The green circle curve represents the Chebyshev AILC-based NN-CFRDPD method (method III). The red curve represents the Gaussian AILC-based NN-CFRDPD method (method IV).

The corresponding ACPR, EVM values, the currents of supply $\left(I_{\text {supply }}\right)$ and PA power added efficiencies (PAE) are given in Table V. The EVM in this paper is calculated with the difference between the I/Q samples of measured signal and 
TABLE V

COMPARISON OF DIFFERENT METHODS WITH TEST BENCH 1

\begin{tabular}{|c|c|c|c|c|c|c|c|c|}
\hline & & PA out & $\mathrm{O}$ & I & II & III & IV & $\mathrm{V}$ \\
\hline \multicolumn{2}{|l|}{$P_{\text {out }}(\mathrm{dBm})$} & 27.1 & 24.2 & 26.9 & 27.5 & 27.1 & 27.2 & 26.9 \\
\hline \multicolumn{2}{|l|}{$I_{\text {supply }}(\mathrm{A})$} & 0.64 & 0.63 & 0.64 & 0.64 & 0.64 & 0.64 & 0.64 \\
\hline \multicolumn{2}{|l|}{ PAE (\%) } & 16.0 & 8.4 & 15.3 & 17.6 & 16.0 & 16.4 & 15.3 \\
\hline \multirow{4}{*}{$\begin{array}{l}\text { ACPR } \\
(\mathrm{dBc})\end{array}$} & L1 & -36.3 & -45.0 & -42.4 & -41.0 & -43.6 & -43.2 & -38.9 \\
\hline & $\mathrm{U} 1$ & -36.9 & -45.7 & -43.0 & -41.5 & -44.0 & -43.8 & -38.5 \\
\hline & $\mathrm{L} 2$ & -43.6 & -46.3 & -45.1 & -43.0 & -45.8 & -45.9 & -42.8 \\
\hline & $\mathrm{U} 2$ & -44.2 & -46.8 & -46.0 & -43.1 & -46.2 & -46.4 & -44.3 \\
\hline \multicolumn{2}{|l|}{ EVM (\%) } & 15.3 & 2.4 & 4.5 & 7.0 & 3.6 & 3.6 & 4.6 \\
\hline \multicolumn{2}{|l|}{ Complexity (flops) } & - & 2702 & 166 & 2702 & 2702 & 2702 & 166 \\
\hline
\end{tabular}

O: Only DPD without CFR; I: GMP-CFRDPD in [14]; II: NN-CFRDPD with conventional ILC; III: NN-CFRDPD with Chebyshev AILC; IV: NN-CFRDPD with Gaussian AILC; V: GMP-CFRDPD in [13]

estimated signal [42]:

$$
\mathrm{EVM}_{\%}=\sqrt{\frac{\frac{1}{N} \sum_{j=0}^{N-1}\left(\delta I^{2}+\delta Q^{2}\right)}{S_{\text {avg }}^{2}}} \times 100 \%
$$

where $\delta I$ and $\delta Q$ are errors magnitude corresponding to inphase symbol and quadrature symbol of linearized signal samples compared with the desired signal samples respectively, $N$ is the number of samples, $S_{\text {avg }}^{2}$ is the average square magnitude of $N$ samples. The result of method O (only DPD without (FF) is given as a reference.

Compared with method $\mathrm{O}$, the proposed NN-CFRDPD which reduces the PAPR of the input signal of $3 \mathrm{~dB}$ can improve the PAE from $8.4 \%$ to at least $16.0 \%$. The proposed NN-CFRDPD achieves around $-44 \mathrm{dBc}$ on ACPR, which trades $1 \mathrm{~dB}$ off compared with only DPD linearization to double the PAE.

Compared with method II and method V, method III and IV have better linearization performance in ACPR and EVM. The NN-CFRDPD based on Gaussian window and Chebyshev AILC exhibit similar performance. Both of them improve the ACPR 2-3 dB and the EVM is halved. Comparing with GMPbased joint CFR/DPD in literature (method I), our proposed NN-CFRDPD can reach similar performance while using Chebyshev AILC and shows $1 \mathrm{~dB}$ improvement on ACPR. The NN-CFRDPD based on AILC can outperform the one based on conventional ILC because the proposed AILC tackles the problem of convergence caused by filtered errors and thus gives a better target signal for $\mathrm{NN}$ training. The NN model shows a very strong modeling accuracy compared with conventional Volterra-series-based models, which therefore exhibits much dependence on the training signals.

The complexity of NN-CFRDPD seems higher than GMPCFRDPD in Table V. That is because the GMP model structure is determined by the algorithm in [41] which optimizes both the model modeling accuracy and model complexity. On the other hand, the hyperparameters of NN model are determined by the algorithm in [21] which considers only average modeling accuracy since $\mathrm{NN}$ training signals are randomly partitioned and the corresponding performance is not a constant value for the same group of hyperparameters. Furthermore, we can see that the proposed AILC-based NNCFRDPD reaches at least $1 \mathrm{~dB}$ better than the GMP-CFRDPD.

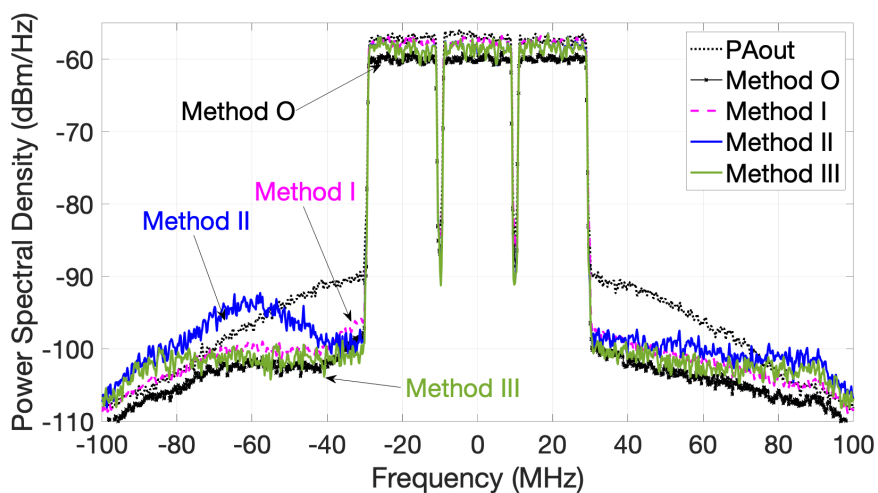

O: Only DPD without CFR; I: GMP-CFRDPD in [14]; II: NN-CFRDPD with conventional ILC; III: NN-CFRDPD with Chebyshev AILC

Fig. 21. PA output spectra without and with only DPD, and with NNCFRDPD on Test bench 2

TABLE VI

COMPARISON OF DIFFERENT METHODS WITH TEST BENCH 2

\begin{tabular}{|c|c|c|c|c|c|c|}
\hline \multicolumn{2}{|c|}{} & PA out & O & I & II & III \\
\hline \multicolumn{2}{|c|}{$P_{\text {out }}(\mathrm{dBm})$} & 31.5 & 29.8 & 31.5 & 31.6 & 31.6 \\
\hline \multicolumn{2}{|c|}{$I_{\text {supply }}(\mathrm{A})$} & 0.10 & 0.10 & 0.10 & 0.10 & 0.10 \\
\hline \multicolumn{2}{|c|}{ PAE (\%) } & 70.6 & 47.8 & 70.6 & 72.3 & 72.3 \\
\hline ACPR & L1 & -36.0 & -45.0 & -43.0 & -37.9 & -45.7 \\
\cline { 2 - 7 } & U1 & -35.1 & -45.8 & -43.8 & -41.5 & -46.2 \\
\hline \multicolumn{2}{|c|}{ EVM (\%) } & 18.1 & 2.7 & 5.3 & 6.5 & 4.9 \\
\hline \multicolumn{2}{|c|}{ Complexity (flops) } & - & 1283 & 262 & 1283 & 1283 \\
\hline
\end{tabular}

O: Only DPD without CFR; I: GMP-CFRDPD in [14]; II: NN-CFRDPD with conventional ILC; III: NN-CFRDPD with Chebyshev AILC

We also test these approaches with Test bench 2 using the stimulus with wider band on a more powerful PA. The GMPCFRDPD model for method I is given as

$$
\begin{aligned}
\mathcal{K}_{a} & =3, \mathcal{L}_{a}=6 \\
\mathcal{K}_{b} & =1, \mathcal{L}_{b}=1, \mathcal{M}_{b}=1 \\
\mathcal{K}_{c} & =1, \mathcal{L}_{c}=7, \mathcal{M}_{c}=2 .
\end{aligned}
$$

with 33 coefficients. We set NN model with time delay $L=9$ and $N_{1}=18, N_{2}=15$ neurons for all tests.

The spectra of different approaches are given in Fig. 21. The gray dotted curve is the PA output without DPD linearization. The black point curve stands for the PA output linearized by only NN DPD (method O). The pink dashed curve represents the GMP-based joint CFR/DPD in [14] (method I). The blue curve represents the conventional-ILC-based NN-CFRDPD 
method (method II). The green curve represents the Chebyshev AILC-based NN-CFRDPD method (method III). Gaussian AILC is not presented according to former results.

The power efficiencies are given in Table VI as well as the corresponding linearization performance and complexity. Using the same model, the joint CFR/DPD improves the PA efficiency over $20 \%$ for a similar linearization performance without adding complexity for the CFR.

Comparing with method I, the proposed method III has $2 \sim 3 \mathrm{~dB}$ advantage on ACPR, which provides more margin of linearity to enhance the PA efficiency. Though the complexity of NN model is higher than that of GMP, it offers an alternative option which results in a different trade-off between linearity and complexity, or a different trade-off between saved PA power and the power consumed by DSP.

Comparing with method II based on conventional ILC, the proposed method III has over $5 \mathrm{~dB}$ advantage on ACPR. The spectra of method II in Fig. 21 shows the impact of conventional ILC on out-of-band which can be eliminated by the proposed AILC as shown by the spectra of method III.

The experimental results show that the proposed NNCFRDPD can keep a similar linearization performance as a single DPD while largely enhancing the PA efficiency by incorporating the CFR. The proposed AILC is crucial to implement the NN-CFRDPD for high PA efficiency with an acceptable linearization performance.

\section{CONCLUSION}

In this paper, we propose an NN-based joint CFR/DPD to enhance the PA power efficiency while keeping a good linearization performance. Compared with joint CFR/DPD based on Volterra series, e.g. GMP, the proposed NN-CFRDPD has higher complexity but better linearization performance. It is more an alternative option depending on the vision of the trade-off between the power saved from the PA and the power consumed by the joint CFR/DPD itself, rather than a substitution of the GMP-based joint CFR/DPD in [14]. With low power-consuming digital circuits developing recently, the proposed NN-CFRDPD is promising. In the future work, an algorithm should be developed to reduce the complexity of NN model in the same way as the algorithm in [41]. In order to achieve the best performance of the NN model, we also propose an AILC algorithm in this paper to compute the target signal used for training. Different types of windows are applied in AILC to compensate for the out-of-band distortion brought in conventional ILC by the iteratively filtered white noise at the PA input. Experimental results confirm that the proposed approach has good linearization performance while exhibiting a high efficiency.

\section{ACKNOWLEDGMENT}

The authors would like to thank T. Eriksson, and C. Fager, Chalmers University of Technology, for their permission to use the RF WebLab measurement setup.

\section{REFERENCES}

[1] L. Guan and A. Zhu, "Green communications: Digital predistortion for wideband rf power amplifiers," IEEE Microw. Mag., vol. 15, no. 7, pp. 84-99, Nov 2014.

[2] F. M. Ghannouchi, "Power amplifier and transmitter architectures for software defined radio systems," IEEE Circuits Syst. Mag., vol. 10, no. 4, pp. 56-63, Fourthquarter 2010.

[3] A. Katz, J. Wood, and D. Chokola, "The evolution of pa linearization: From classic feedforward and feedback through analog and digital predistortion," IEEE Microw. Mag., vol. 17, no. 2, pp. 32-40, Feb 2016.

[4] J. Chani-Cahuana, P. N. Landin, C. Fager, and T. Eriksson, "Iterative learning control for of power amplifier linearization," IEEE Trans. Microw. Theory Techn., vol. 64, no. 9, pp. 2778-2789, Sep. 2016.

[5] J. Kim and K. Konstantinou, "Digital predistortion of wideband signals based on power amplifier model with memory," Electronics Letters, vol. 37, no. 23, pp. 1417-1418, Nov 2001.

[6] D. Morgan, Z. Ma, J. Kim, M. Zierdt, and J. Pastalan, "A generalized memory polynomial model for digital predistortion of rf power amplifiers," IEEE Trans. Signal Process., vol. 54, no. 10, pp. 3852-3860, Oct. 2006.

[7] A. Zhu, J. Pedro, and T. Brazil, "Dynamic deviation reduction-based volterra behavioral modeling of rf power amplifiers," IEEE Trans. Microw. Theory Techn., vol. 54, no. 12, pp. 4323-4332, Dec 2006.

[8] S. Wang, M. Abi Hussein, O. Venard, and G. Baudoin, "Optimal sizing of two-stage cascaded sparse memory polynomial model for high power amplifiers linearization," IEEE Trans. Microw. Theory Techn., vol. 66, no. 9, pp. 3958-3965, Sep. 2018.

[9] J. Wood, Behavioral Modeling and Linearization of RF Power Amplifiers:, ser. Artech House Microwave Library. Artech House, 2014.

[10] S. Wang, M. Roger, and C. Lelandais-Perrault, "Impacts of crest factor reduction and digital predistortion on linearity and power efficiency of power amplifiers," IEEE Trans. Circuits Syst. II, Exp. Briefs, vol. 66, no. 3, pp. 407-411, Mar. 2019.

[11] H. Enzinger, K. Freiberger, and C. Vogel, "Competitive linearity for envelope tracking: Dual-band crest factor reduction and 2d-vectorswitched digital predistortion," IEEE Microw. Mag., vol. 19, no. 1, pp. 69-77, Jan. 2018.

[12] M. V. Amiri, M. Helaoui, N. Boulejfen, and F. M. Ghannouchi, "Optimized spectrum constrained crest factor reduction technique using polynomials," IEEE Trans. Commun., vol. 63, no. 7, pp. 2555-2564, July 2015.

[13] R. N. Braithwaite, "A combined approach to digital predistortion and crest factor reduction for the linearization of an rf power amplifier," IEEE Trans. Microw. Theory Techn., vol. 61, no. 1, pp. 291-302, Jan. 2013.

[14] S. Wang, M. Roger, J. Sarrazin, and C. Lelandais-Perrault, "A joint crest factor reduction and digital predistortion for power amplifiers linearization based on clipping-and-bank-filtering," IEEE Trans. Microw. Theory Techn., pp. 1-9, Dec. 2019.

[15] K. Samdanis and T. Taleb, "The road beyond 5g: A vision and insight of the key technologies," IEEE Network, pp. 1-7, 2020.

[16] S. Duan, X. Hu, Z. Dong, L. Wang, and P. Mazumder, "Memristor-based cellular nonlinear/neural network: Design, analysis, and applications," IEEE Trans. Neural Netw. Learn. Syst., vol. 26, no. 6, pp. 1202-1213, 2015.

[17] F. Cai, J. M. Correll, S. H. Lee, Y. Lim, V. Bothra, Z. Zhang, M. P. Flynn, and W. D. Lu, "A fully integrated reprogrammable memristor cmos system for efficient multiply-accumulate operations," Nature Electronics, vol. 2, no. 7, pp. 290-299, Jul. 2019.

[18] Qi-Jun Zhang, K. C. Gupta, and V. K. Devabhaktuni, "Artificial neural networks for rf and microwave design - from theory to practice," IEEE Trans. Microw. Theory Techn., vol. 51, no. 4, pp. 1339-1350, Apr. 2003.

[19] M. Rawat, K. Rawat, and F. Ghannouchi, "Adaptive digital predistortion of wireless power amplifiers/transmitters using dynamic real-valued focused time-delay line neural networks," IEEE Trans. Microw. Theory Techn., vol. 58, no. 1, pp. $95-104$, jan. 2010.

[20] F. Mkadem and S. Boumaiza, "Physically inspired neural network model for rf power amplifier behavioral modeling and digital predistortion," IEEE Trans. Microw. Theory Techn., vol. 59, no. 4, pp. 913-923, Apr. 2011.

[21] S. Wang, M. Roger, J. Sarrazin, and C. Lelandais-Perrault, "Hyperparameter optimization of two-hidden-layer neural networks for power amplifiers behavioral modeling using genetic algorithms," IEEE Microw. Wireless Compon. Lett., vol. 29, no. 12, pp. 802-805, Dec. 2019. 
[22] M. Rawat and F. Ghannouchi, "Distributed spatiotemporal neural network for nonlinear dynamic transmitter modeling and adaptive digital predistortion," IEEE Trans. Instrum. Meas., vol. 61, no. 3, pp. 595 -608, march 2012.

[23] M. Li, J. Liu, Y. Jiang, and W. Feng, "Complex-chebyshev functional link neural network behavioral model for broadband wireless power amplifiers," IEEE Trans. Microw. Theory Techn., vol. 60, no. 6, pp. 1979 -1989 , june 2012.

[24] M. Rawat and F. M. Ghannouchi, "A mutual distortion and impairment compensator for wideband direct-conversion transmitters using neural networks," IEEE Trans. Broadcast., vol. 58, no. 2, pp. 168-177, June 2012.

[25] P. Jaraut, M. Rawat, and F. M. Ghannouchi, "Composite neural network digital predistortion model for joint mitigation of crosstalk,i/q imbalance, nonlinearity in mimo transmitters," IEEE Trans. Microw. Theory Techn., vol. 66, no. 11, pp. 5011-5020, Nov. 2018.

[26] I. Sohn and S. C. Kim, "Neural network based simplified clipping and filtering technique for papr reduction of ofdm signals," IEEE Commun. Lett., vol. 19, no. 8, pp. 1438-1441, Aug. 2015.

[27] M. Abi Hussein, V. Bohara, and O. Venard, "On the system level convergence of ila and dla for digital predistortion," in Wireless Communication Systems (ISWCS), 2012 International Symposium on, Aug 2012, pp. 870-874.

[28] S. Wang, M. Roger, and C. Lelandais-Perrault, "Clipping-and-bankfiltering technique in joint crest factor reduction and digital predistortion for power amplifiers," in 2018 Asia-Pacific Microwave Conference (APMC), Nov. 2018, pp. 768-770.

[29] K. Li, N. Guan, and H. Wang, "Iterative learning control assisted neural network for digital predistortion of mimo power amplifier," in 2018 IEEE 87th Vehicular Technology Conference (VTC Spring), Jun. 2018, pp. $1-5$.

[30] M. Schoukens, J. Hammenecker, and A. Cooman, "Obtaining the preinverse of a power amplifier using iterative learning control," IEEE Trans. Microw. Theory Techn., vol. 65, no. 11, pp. 4266-4273, Nov. 2017.

[31] P. Draxler, J. Deng, D. Kimball, I. Langmore, and P. M. Asbeck, "Memory effect evaluation and predistortion of power amplifiers," in IEEE MTT-S International Microwave Symposium Digest, 2005., 2005, pp. 4 pp.-

[32] S. Wang, M. Abi Hussein, O. Venard, and G. Baudoin, "Impact of the normalization gain of digital predistortion on linearization performance and power added efficiency of the linearized power amplifier," in 2017 12th European Microwave Integrated Circuits Conference (EuMIC), Oct. 2017, pp. 310-313.

[33] T. Liu, S. Boumaiza, and F. Ghannouchi, "Deembedding static nonlinearities and accurately identifying and modeling memory effects in wide-band rf transmitters," IEEE Trans. Microw. Theory Techn., vol. 53, no. 11, pp. 3578-3587, Nov. 2005.

[34] M. Schoukens and Y. Rolain, "Parametric identification of parallel wiener systems," IEEE Trans. Instrum. Meas., vol. 61, no. 10, pp. 28252832, Oct. 2012.

[35] M. Hussein, V. Bohara, and O. Venard, "Multi-stage digital predistortion based on indirect learning architecture," in Acoustics, Speech and Signal Processing (ICASSP), 2013 IEEE International Conference on, May 2013, pp. 6093-6097.

[36] K. Anoh, C. Tanriover, and B. Adebisi, "On the optimization of iterative clipping and filtering for papr reduction in ofdm systems," IEEE Access, vol. 5, pp. 12004-12 013, 2017.

[37] R. Hongyo, Y. Egashira, T. M. Hone, and K. Yamaguchi, "Deep neural network-based digital predistorter for doherty power amplifiers," IEEE Microw. Wireless Compon. Lett., vol. 29, no. 2, pp. 146-148, Feb. 2019.

[38] M. T. Hagan and M. B. Menhaj, "Training feedforward networks with the marquardt algorithm," IEEE Trans. Neural Netw., vol. 5, no. 6, pp. 989-993, Nov. 1994.

[39] P. N. Landin, S. Gustafsson, C. Fager, and T. Eriksson, "Weblab: A webbased setup for pa digital predistortion and characterization [application notes]," IEEE Microw. Mag., vol. 16, no. 1, pp. 138-140, Feb 2015.

[40] A. S. Tehrani, H. Cao, S. Afsardoost, T. Eriksson, M. Isaksson, and C. Fager, "A comparative analysis of the complexity/accuracy tradeoff in power amplifier behavioral models," IEEE Trans. Microw. Theory Techn., vol. 58, no. 6, pp. 1510-1520, June 2010.

[41] S. Wang, M. Abi Hussein, O. Venard, and G. Baudoin, "A novel algorithm for determining the structure of digital predistortion models," IEEE Trans. Veh. Technol., vol. 67, no. 8, pp. 7326-7340, Aug. 2018.

[42] M. Isaksson, D. Wisell, and D. Ronnow, "A comparative analysis of behavioral models for rf power amplifiers," IEEE Trans. Microw. Theory Techn., vol. 54, no. 1, pp. 348-359, Jan. 2006

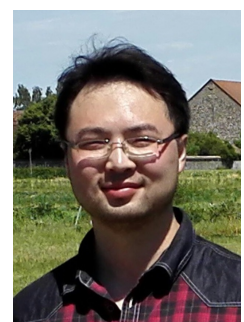

Siqi Wang (M'20) received the B.S. degree from Huazhong University of Science and Technology, Wuhan, China in 2012, the M.S. degree from the University of Paris-Sud, Orsay, France in 2014, and the Ph.D. degree from the University of Paris-Est Marne La Vallée, Champs sur Marne, France, in 2018. He has been a post-doc fellow with GeePs, Centralesupélec in 2018-2019. He is currently a post-doc fellow with Chalmers University of Technology. His research interests include wireless communications, signal processing, digital predistortion, energy efficiency optimization for wireless communication systems.

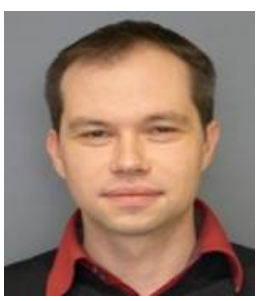

Morgan Roger (M'07) graduated from the Ecole Spéciale des Travaux Publics in Paris in 2002, with an emphasis on mechanics, electrical engineering and electronics. The same year, he joined the Department of Electronic Systems of CentraleSupélec in Gif-sur-Yvette to pursue his Ph.D. in signal processing. He received it from the Paris-Sud University (Orsay) in 2007 and is now an associate professor of CentraleSupélec, affiliated with the GeePs laboratory. His current research interests include data converters and signal processing methods for electronic systems with an emphasis on power amplifier linearization. He is an IEEE member.

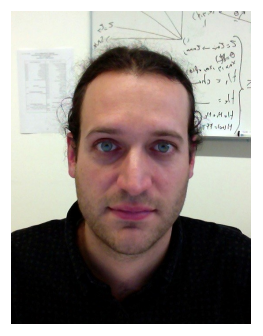

Julien Sarrazin (S'05-M'10-SM'19) received his Engineering diploma/Master of Research, and $\mathrm{PhD}$ degrees from the University of Nantes in France, in 2005 and 2008 respectively. In 2009 and 2010 he worked at the BK Birla Institute of Technology of Pilani, in India, where he was in charge of telecommunication-related teaching. In 2011 and 2012, he was a research engineer at Telecom ParisTech in Paris. Since September 2012, he is an Associate Professor at Sorbonne Université (formerly University of Pierre and Marie Curie) in Paris, where he is currently working in the GEEPS research institute (Group of Electrical Engineering of Paris) in the field of Spatial Data Focusing, antenna design, and localization. His research interests also include channel modeling for Body Area Networks. He is an IEEE senior member and reviewer and has published over 110 technical journals, patents, book chapters, and conference papers.

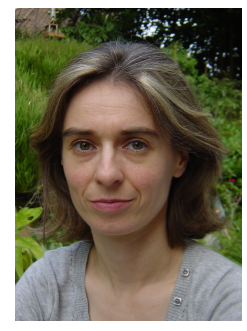

Caroline Lelandais-Perrault received in 1995 the diploma from the Ecole Supérieure d'Electricité (Supélec), one of the French Grandes Ecoles. She worked six years as a development engineer and project manager at IN-SNEC (Zodiac Data Systems), a company specialized in telemetry equipments for satellites and Ariane rocket. In 2001, she joined the Electronic System Department at Supélec and received her $\mathrm{PhD}$ in 2006 from Université Paris XI. She is currently Associate Professor in the Dep. of Electronic Systems of CentraleSupélec at Université Paris-Saclay. With GeePs lab. (UMR CNRS 8507), her research activities concern wide-band and versatile and also high resolution analog-to-digital conversion and focus especially on calibration techniques of that systems. 